\title{
Gefitinib loaded folate decorated bovine serum albumin conjugated carboxymethyl-beta- cyclodextrin nanoparticles enhance drug delivery and attenuate autophagy in folate receptor-positive cancer cells
}

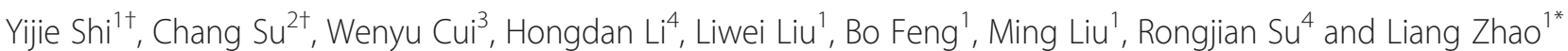

\begin{abstract}
Background: Active targeting endocytosis mediated by the specific interaction between folic acid and its receptor has been a hotspot in biological therapy of many human cancers. Various studies have demonstrated that folate and its conjugates could facilitate the chemotherapeutic drug delivery into folate receptor (FR)-positive tumor cells in vitro and in vivo. In order to utilize FA-FR binding specificity to achieve targeted delivery of drugs into tumor cells, we prepared Gefitinib loaded folate decorated bovine serum albumin conjugated carboxymethyl- $\beta$-cyclodextrin nanoparticles for enhancing drug delivery in cancer cells. On this context, the aim of our study was to develop a novel nano-delivery system for promoting tumor-targeting drug delivery in folate receptor-positive Hela cells.

Results: We prepared folic acid (FA)-decorated bovine serum albumin (BSA) conjugated carboxymethyl- $\beta$-cyclodextrin (CM- $\beta$-CD) nanoparticles (FA-BSA-CM- $\beta-C D$ NPs) capable of entrapping a hydrophobic Gefitinib. It was observed that nanoparticles are monodisperse and spherical nanospheres with an average diameter of $90.2 \mathrm{~nm}$ and negative surface charge of $-18.6 \mathrm{mV}$. FA-BSA-CM- $\beta$-CD NPs could greatly facilitate Gefitinib uptake and enhance the toxicity to folate receptor-positive Hela cells. Under the reaction between FA and FR, Gefitinib loaded FA-BSA-CM- $\beta-C D$ NPs induced apoptosis of Hela cells through elevating the expression of caspase-3 and inhibited autophagy through decreasing the expressing of LC3. It also confirmed that clathrin-mediated endocytosis and macropinocytosis exerted great influence on the internalization of both NPs.
\end{abstract}

Conclusions: These results demonstrated that FA may be an effective targeting molecule and FA-BSA-CM- $\beta$-CD NPs provided a new strategy for the treatment of human cancer cells which over-expressed folate receptors.

Keywords: Folate, Folate receptors, Carboxymethyl- $\beta$-Cyclodextrin, Bovine serum albumin, Nanoparticles, Gefitinib

\section{Background}

Nanosized drug carriers functionalized with moieties specifically targeting tumor cells are promising tools in cancer therapy, due to their ability to circulate in the bloodstream for longer periods and their selectivity for tumor cells, enabling the sparing of healthy tissues [1-5]. Many synthetic biomimetic nanocrystalline apatites are used as nanocarriers to

\footnotetext{
*Correspondence: liangzhao79@163.com

${ }^{\dagger}$ Equal contributors

'College of Pharmacy, Liaoning Medical University, Jinzhou 121000, P R China Full list of author information is available at the end of the article
}

produce multifunctional nanoparticles, by coupling them with the chemotherapeutic drug, such as Gefitinib, Dox or membrane antibody DO-24 monoclonal antibody (mAb) directed against the c-Met/Hepatocyte Growth Factor Receptor (Met/HGFR), which is over-expressed on different kinds of carcinomas and thus represent a useful tumor target recently [6-8]. Gefitinib, a tyrosine kinase inhibitor of Epithelial Growth Factor Receptor (EGFR) usually expressed in solid tumors of epithelial origin, can prevent tumor growth, metastasis and angiogenesis, and promote apoptosis of tumor cells [9-11]. The main mechanism includes that it 
can block the signal transmission by competitive binding Mg-ATP situated on catalytic domain of EGFR-TK, then inhibit the activation of mitogen activated protein kinase, inducing the apoptosis of cancer cells [12]. However, Gefitinib is absorbed slowly and widely distributed in bodies following oral administration, resulting in the serious side effects and lower bioavailability. Moreover, the solubility of Gefitinib is decreased with the decline of $\mathrm{pH}$ in medium [13].

Cyclodextrins (CD), a family of carbohydrate polymers which are produced from starch by enzymatic conversion and commonly used in food, pharmaceutical, drug delivery, and chemical industries, as well as agriculture and environmental engineering, is cyclic oligosaccharide with cone barrel structure composed of seven glucopyranose units with cylindrical cavity [14-16]. The exterior of this cone is polar and hydrophilic, whereas the interior cavity is relatively nonpolar and hydrophobic. Small hydrophobic molecules as the guest molecules can be completely or partially embedded into CD cavity to form complexes, improving water solubility, stability and biological activity of the guest molecules [17-21]. Bovine serum albumin (BSA), a carrier protein, plays an important role in drug storage and transport, for its superior biocompatibility it has been widely used in biomedical research, such as Nano carrier, nanoparticle surface engineering and temples for preparation of nanoparticles [22-25].

To improve the solubility and stability of Gefitinib, we synthesize the amphiphilic BSA-CM- $\beta-C D$ conjugates to prepare the assembled nanoparticles capable of entrapping hydrophobic Gefitinib into the cavity of CD through the host-guest interaction. Folate receptor (FR), as a transmembrane glycoprotein, promotes the transportation of folate (FA) or its conjugates into the cells by active targeting endocytosis mediated through FA-FR interaction [26-28]. FA is expressed at basal levels in normal adult organs such as brain, lung and liver, but it is over-expressed in many human cancers including ovarian cancer, breast cancer, endometrial cancer, lung cancer, kidney cancer, colon cancer and nasopharyngeal carcinoma cells [29-31]. Several lines of evidence have demonstrated that FA and its conjugates could significantly enhance the drug delivery efficiency into FR-positive tumor cells both in vitro and in vivo [32-34]. Herein, FA is adopted as the coupling molecule to improve FR-positive tumor-targeted drug delivery (Figure 1). Properties of NPs such as size, morphology and surface potential were examined. Using Gefitinib as the model drug, we prepared drug-loaded nanoparticles. We found that FA-BSA-CM- $\beta-C D$ NPs greatly facilitated Gefitinib uptake and enhanced the toxic effect in folate receptor-positive Hela cells. Our results demonstrated that FA-BSA-CM- $\beta-C D$ NPs might be a higher efficiency drug delivery system than the conventional delivery system for the targeting therapy of FR positive human cancers.

\section{Results and discussion}

The preparation and characteristics of various kinds of NPs Conjugation of CM- $\beta-C D$ to BSA was obtained by carbodiimide coupling. Carboxylic group of $C M-\beta-C D$ reacted with EDAC to form unstable reactive ester. With addition

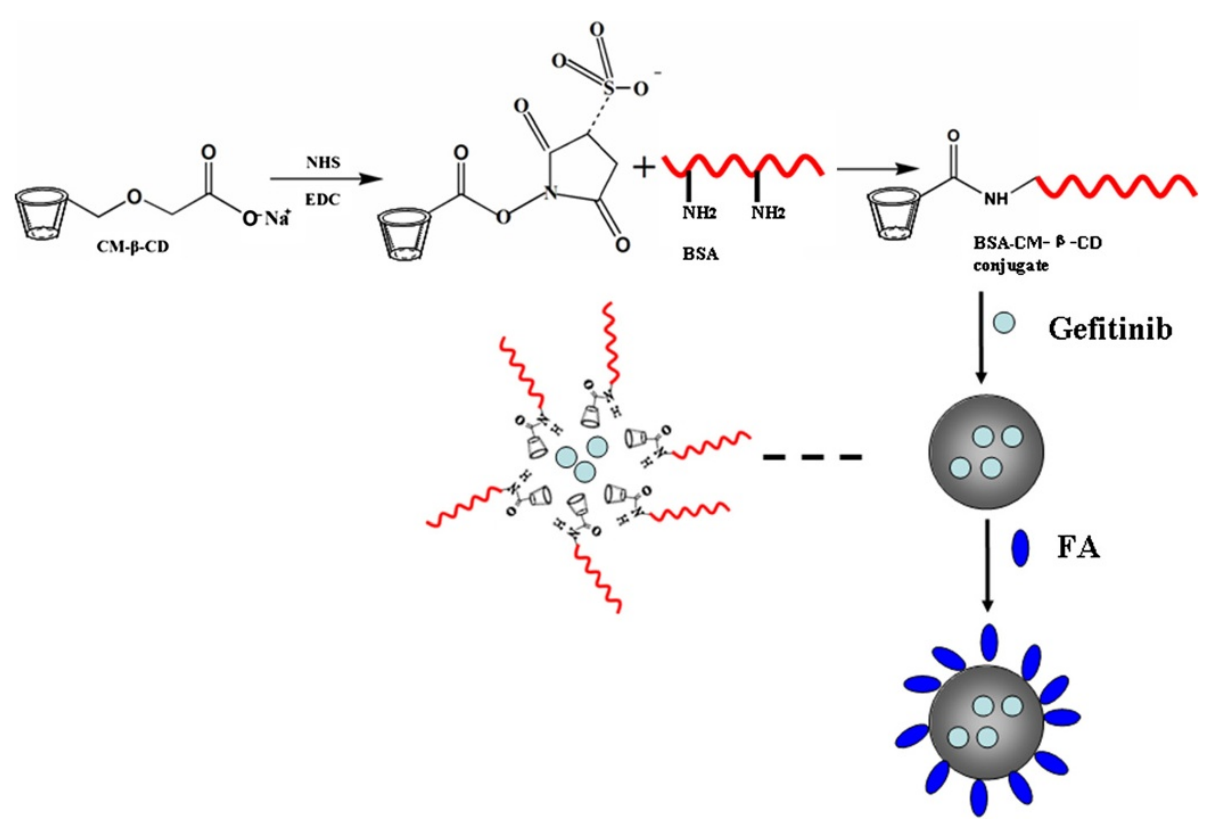

Figure 1 Schematic formation of Gefitinib loaded folate-decorated bovine serum albumin conjugated carboxymethyl- $\beta$-cyclodextrin nanoparticles. 
of NHS, semi-stable amine-reactive NHS-ester was synthesized and then mixed with BSA which containing amino group to obtain CM- $\beta-C D$ conjugated BSA by stable amide bond [35,36].

The characterization of BSA-CM- $\beta-C D$ conjugates was investigated by infrared spectroscopy (Figure 2). The result showed the FT-IR spectra of BSA, CM- $\beta-C D$, and BSA-CM- $\beta-C D$ conjugates. The characteristic peak of BSA-CM- $\beta-C D$ conjugates appeared at $1650 \mathrm{~cm}^{-1}$ and $1540 \mathrm{~cm}^{-1}$ should be ascribed to the newly formed amide bond between $C M-\beta-C D$ molecules and BSA. These data supported that $C M-\beta-C D$ has grafted to BSA, and they are correspond to the results of former literatures [37].

Spectrum of infrared absorption of Gefitinib loaded FA-BSA-CM- $\beta-C D$ NPs was shown in Figure 3. It can be seen that when BSA-CM- $\beta-C D$ NPs was bonded with FA, its spectrum demonstrated that the aromatic amine groups $\left(\mathrm{vNH}_{2}, 3415 \mathrm{~cm}^{-1}\right.$ and $\left.3323 \mathrm{~cm}^{-1}\right)$ from FA disappeared, suggesting that amine groups from FA reacted with carboxylic group of BSA. Furthermore, the characteristic peak of secondary amine group $\left(v \mathrm{NH}, 3398 \mathrm{~cm}^{-1}\right)$ from Gefitinib disappeared because of the encapsulation of Gefitinib into the core of NPs.

\section{Development and properties of Gefitinib-loaded FA-BSA- CM- $\beta$-CD NPs}

Using transmission electronic microscope (TEM), we observed that Gefitinib-loaded FA-BSA-CM- $\beta-C D$ NPs we prepared were monodisperse spheres, and further analysis revealed that the diameters of NPs ranged from 52.1 to $105.6 \mathrm{~nm}$ (Figure 4). Table 1 summarized the average diameters measured by dynamic light scattering (DLS) and surface charge information of these prepared nanoparticles. It was remarkable that Gefitinib-loaded FA-BSA-CM- $\beta-C D$ NPs showed smaller particle size, negative zeta potential. The average encapsulation efficiency of Gefitinib in FA-BSA-CM- $\beta$-CD NPs was $89.2 \%$ and about $70.1 \%$ of FA was conjugated on the surface of NPs.

\section{In vitro drug release study}

Gefitinib loaded FA-BSA-CM- $\beta-C D$ NPs exhibited similar release profiles in the medium with different $\mathrm{pH}$. The release curve in PBS could be divided into two phases: initial fast drug release stage and later stable release stage. Gefitinib was released rapidly in the initial fast release stage, and was released slowly in the later stable stage through diffusion because of the continuous degradation of the polymer (Figure 5). The release speed of Gefitinib decreased with the increase of $\mathrm{pH}$ as polymer degraded faster in acid medium. Thus, it possibly suggested that the matrix of NPs tended to be eroded as a result of depolymerization of BSA at $\mathrm{pH} 5.0$ which was closer to the isoelectric points of BSA ( $\mathrm{pH} 4.9$ ), and then drug could be more easily released from NPs. The release ratio during the first $48 \mathrm{~h}$ accounted for over $40 \%$ of the total drug and the remnants were released over longer time of incubation. It suggested that Gefitinibloaded FA-BSA-CM- $\beta$-CD NPs could be used as a longlasting and effective drug delivery system.

\section{Cell viability assays}

The cytotoxic effects of Gefitinib loaded FA-BSA-CM- $\beta-C D$ NPs and BSA-CM- $\beta-C D$ NPs were evaluated by MTT assay using Hela cell line. MTT analysis showed that in the absence of FA in culturing medium, treatment of Hela cells with Gefitinib loaded FA-BSA-CM- $\beta-C D$ NPs caused a markedly increase in the cell cytotoxic activities as compared with free Gefitinib and Gefitinib loaded FA unconjugated NPs (Figure 6B). The $\mathrm{IC}_{50}$ values of Gefitinib loaded FA-BSA-CM- $\beta$-CD NPs treated Hela cells was $4.63 \mu \mathrm{g} / \mathrm{mL}$, $7.85 \mu \mathrm{g} / \mathrm{mL}$ for free Gefitinib and $13.55 \mu \mathrm{g} / \mathrm{mL}$ for Gefitinib loaded BSA-CM- $\beta$-CD NPs. However, no obvious cytotoxic activities were observed when treating the cells with blank FA-BSA-CM- $\beta-C D$ NPs in Hela cells (Figure 6A). These

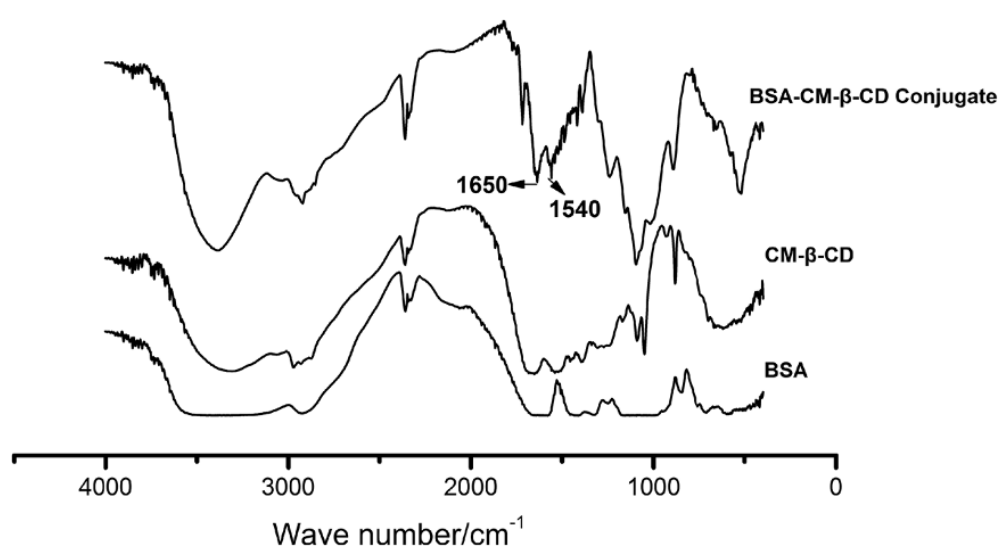

Figure 2 FT-IR spectra of BSA, CM- $\beta-C D$, and BSA-CM- $\beta-C D$ conjugates. 


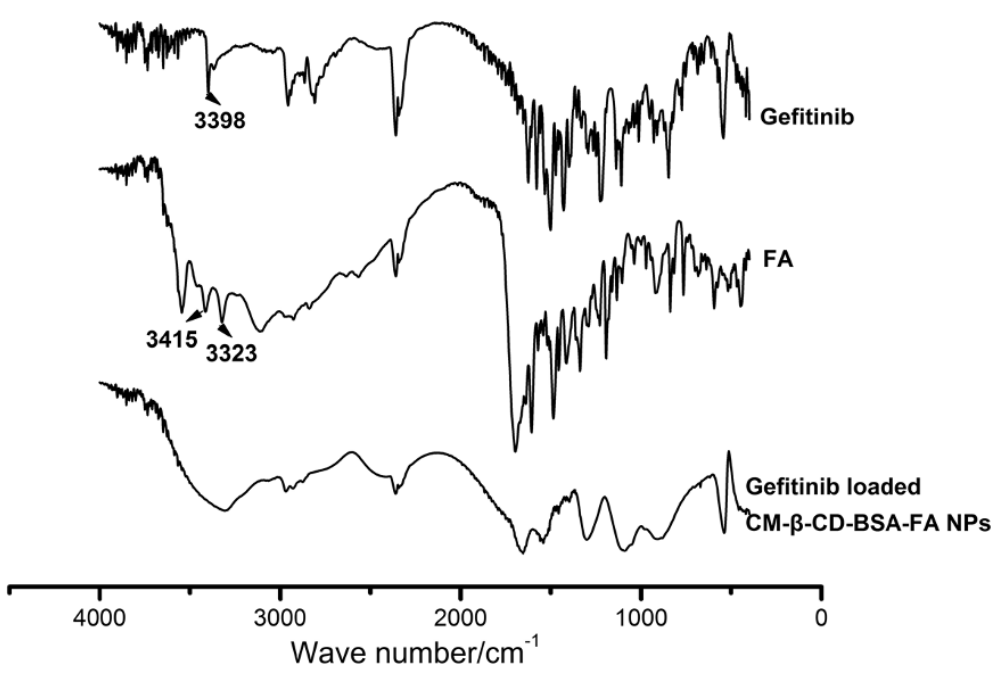

Figure 3 FT-IR spectra of FA, Gefitinib and Gefitinib loaded FA-BSA-CM- $\beta$-CD NPs.

data suggested that more drug loaded FA conjugated NPs could be internalized into Hela cells which expressed FA at higher level by the interaction between FA and FR, further leading to the significant cytotoxicity by the accumulation of drug in cells.

We next examined whether inhibition of FR, which expressed on the cell surface, affected the cytotoxic activities of Gefitinib loaded FA-BSA-CM- $\beta-C D$ NPs. Hela cells were cultured in the medium containing $\mathrm{FA}$ at $5 \mu \mathrm{g} / \mathrm{mL}$. MTT analysis (Figure $6 \mathrm{C}$ ) revealed that the presence of FA caused a significant decrease in the cytotoxic effect of Gefitinib loaded FA-BSA-CM- $\beta-C D$ NPs compared with that without FA in Hela cells. However, pretreatment of FA had little effect on the cytotoxic activity of free Gefitinib and Gefitinib loaded BSA-CM- $\beta-C D$ NPs. The $\mathrm{IC}_{50}$ values of Gefitinib loaded FA-BSA-CM- $\beta$ -
CD NPs treated Hela cells was $13.02 \mu \mathrm{g} / \mathrm{mL}, 8.63 \mu \mathrm{g} / \mathrm{mL}$ for free Gefitinib and $14.76 \mu \mathrm{g} / \mathrm{mL}$ for Gefitinib loaded BSA-CM- $\beta-C D$ NPs. These data further demonstrated that FA conjugation played critical roles in accumulating NPs inside FR-positive tumor cells and could be used as a targeting molecule in the treatment of human cancers which over-expressed FR on the cell surface.

\section{In vitro uptake ability analysis}

To visualize whether FA conjugation could enhance the uptake of BSA-CM- $\beta-C D$ NPs, The NPs were labeled with Rodamine $B$ and the uptake ability was evaluated in Hela cells. Using confocal laser scanning microscopy analysis, Hela cells showed increased number of red fluorescence patches in the cytoplasm when incubating Rhodamine B-labeled FA-BSA-CM- $\beta$-CD NPs in the
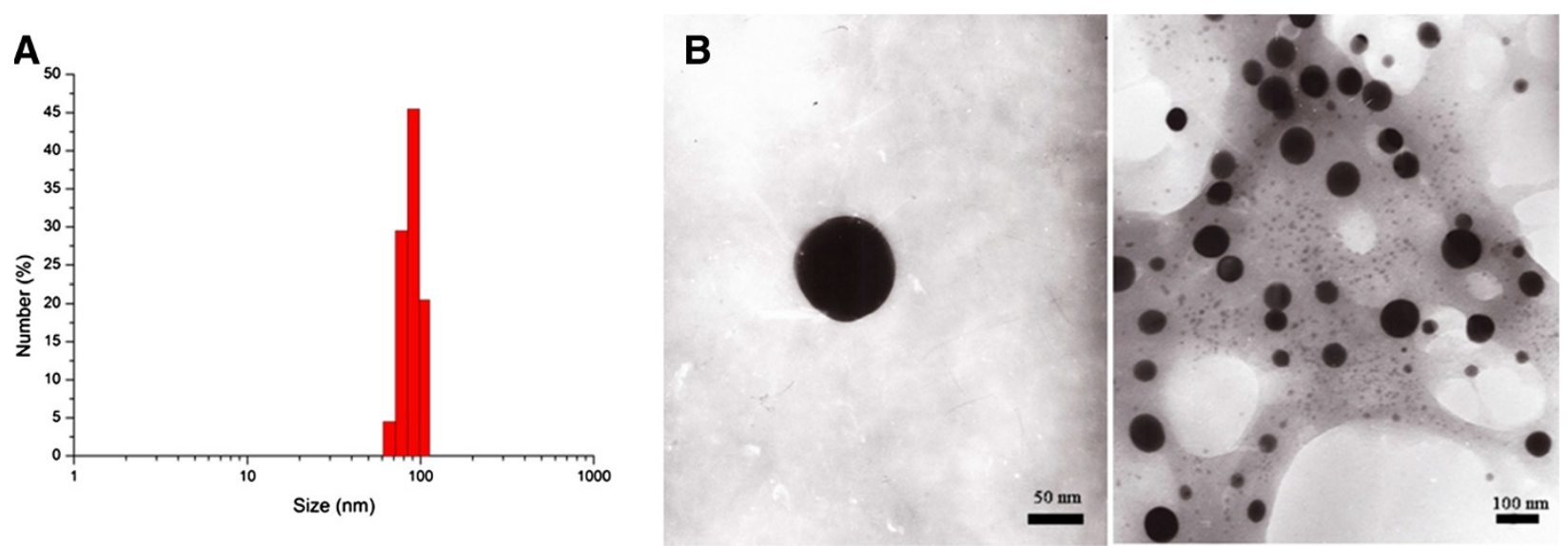

Figure 4 Particle size distribution (A) and TEM image (B) of the obtained Gefitinib-loaded FA-BSA-CM- $\beta$-CD NPs. 
Table 1 Key parameters of Gefitinib-loaded FA-BSA-CM- $\beta$ CD NPs

\begin{tabular}{ll}
\hline Parameters & Data \\
\hline Average diameter (nm) & $90.2 \pm 10.01$ \\
Zeta potential (mv) & $-18.6 \pm 5.8$ \\
Encapsulating efficiency of Gefitinib (\%) & $89.2 \pm 4.7$ \\
Entrapment efficiency of FA (\%) & $70.1 \pm 4.3$ \\
Polydispersity Index (PDI) & 0.093 \\
\hline
\end{tabular}

FA-free medium for $6 \mathrm{~h}$ compared with that of Rhodamine B-labeled BSA-CM- $\beta$-CD NPs. However, the uptake of FA-BSA-CM- $\beta-C D$ NPs was significantly reduced by addition of FA in the medium (Figure 7). The free FA competition study suggested that free FA in medium competed to bind FR on the surface of Hela cells with FA conjugated NPs, leading to the lower uptake of NPs.

\section{Intracellular ATP level assay}

After cells were treated with free Gefitinib, Gefitinib loaded BSA-CM- $\beta$-CD NPs, Gefitinib loaded FA-BSACM- $\beta$-CD NPs, the changing rates of intracellular ATP level were presented in Figure 8. It can be seen that compared with ATP level of untreated Hela cells as the control group, The changing rates of intracellular ATP level for free Gefitinib, Gefitinib loaded BSA-CM- $\beta-C D$ NPs and Gefitinib loaded FA-BSA-CM- $\beta$-CD NPs were decreased to $70.5 \%, 75.4 \%$ and $50.1 \%$, respectively. The results showed that Gefitinib and Gefitinib loaded NPs were internalized to induce the apoptosis of cells by lowering ATP level rates. It also confirmed that with the interaction between FA conjugated on the surface of

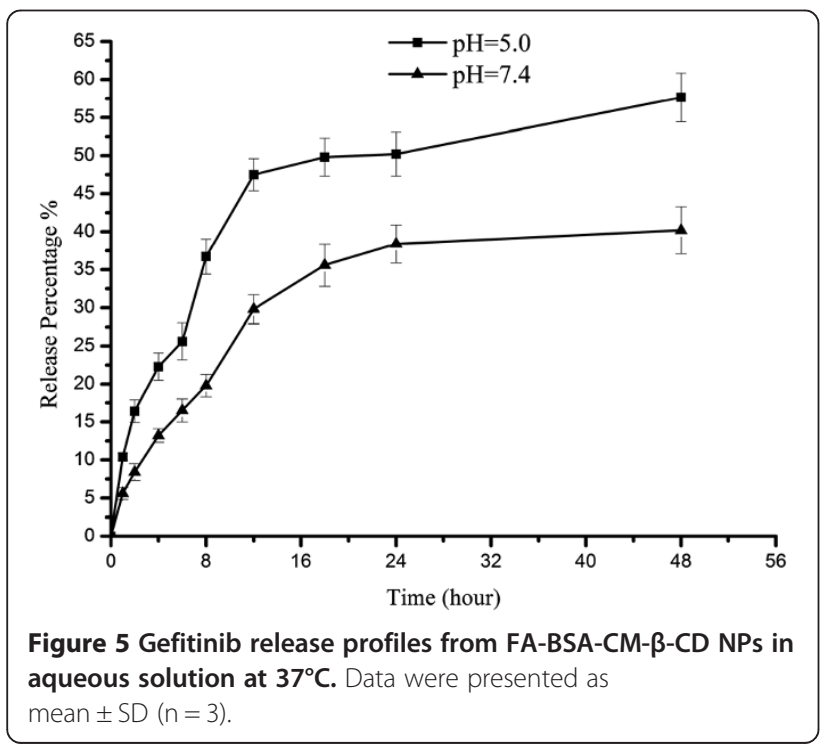

NPs and FR situated at Hela cells, more drug loaded FA conjugated NPs were transported into the interior of cells to inhibit energy generation and accelerate the apoptosis of cells by accumulation of drugs in cells.

\section{Cell apoptosis analysis}

To identify the effect of Gefitinib and Gefitinib loaded NPs on cell apoptosis and autophagy, we detected the expression of caspase-3, Bax, and LC3 by western blot (Figure 9). Compared with free Gefitinib and Gefitinib loaded NPs, Gefitinib loaded FA-BSA-CM- $\beta$-CD NPs induced the highest caspase-3 protein expression. It also illustrated that with the mediation of FA, a large amount of drug loaded FA conjugated NPs were accumulated in Hela cells and caspase- 3 as the main apoptosis relevant protein was increased, corresponding with the results of MTT experiments. However, there was no obvious difference on the Bax protein expression in the treated groups and the control group, confirming that Bax was not involved in Gefitinib induced cell apoptosis (Figure 9A). LC3 (microtubule-associated protein light chain 3) is a specific autophagic marker in mammalian cells during autophagy. So, to identify whether Gefitinib affects autophagy, expression of LC3 was detected in Hela cells, and found that free Gefitinib did not influence the expression of LC3, but with the addition of NPs, the expression of LC3 has been inhibited, also, with the mediation of FA, the inhibition rate increased obviously (Figure 9B). So, the results suggested that through autophagy, Hela cells may be survival and resist free Gefitinib, and FA-NPs mediated accumulation of Gefitinib in cells inhibits LC3 expression. Taken together, through inhibition of autophagy, Gefitinib loaded FA-BSA-CM- $\beta-C D$ NPs induced cells apoptosis.

\section{Inhibition of various endocytosis assay}

To get more insight to know which uptake mechanisms were implied in NPs uptake, Hela cells were pretreated with various endocytic inhibitors specific for a particular endocytic pathway. Figure 10 showed that when genistein as an inhibitor to block caveolae-mediated endocytosis (CvME) was added into cells, there was no significant difference in both NPs internalization suggesting a minor role of CvME. When cells were treated with cytochalasin $\mathrm{D}(30 \mu \mathrm{M}$, macropinocytosis), the uptake ability of both NPs were significantly decreased to $55.4 \%$ and $60.2 \%$. It was also observed that internalization of both NPs in cells with chlorpromazine treatment (clathrin-mediated endocytosis) was significant lower than that in untreated cells. Moreover, $40.1 \%$ reduction in FA-BSA-CM- $\beta-C D$ NPs was observed in comparison with $32.1 \%$ reduction of intracellular uptake of BSA-CM- $\beta$-CD NPs. Some previous study have 

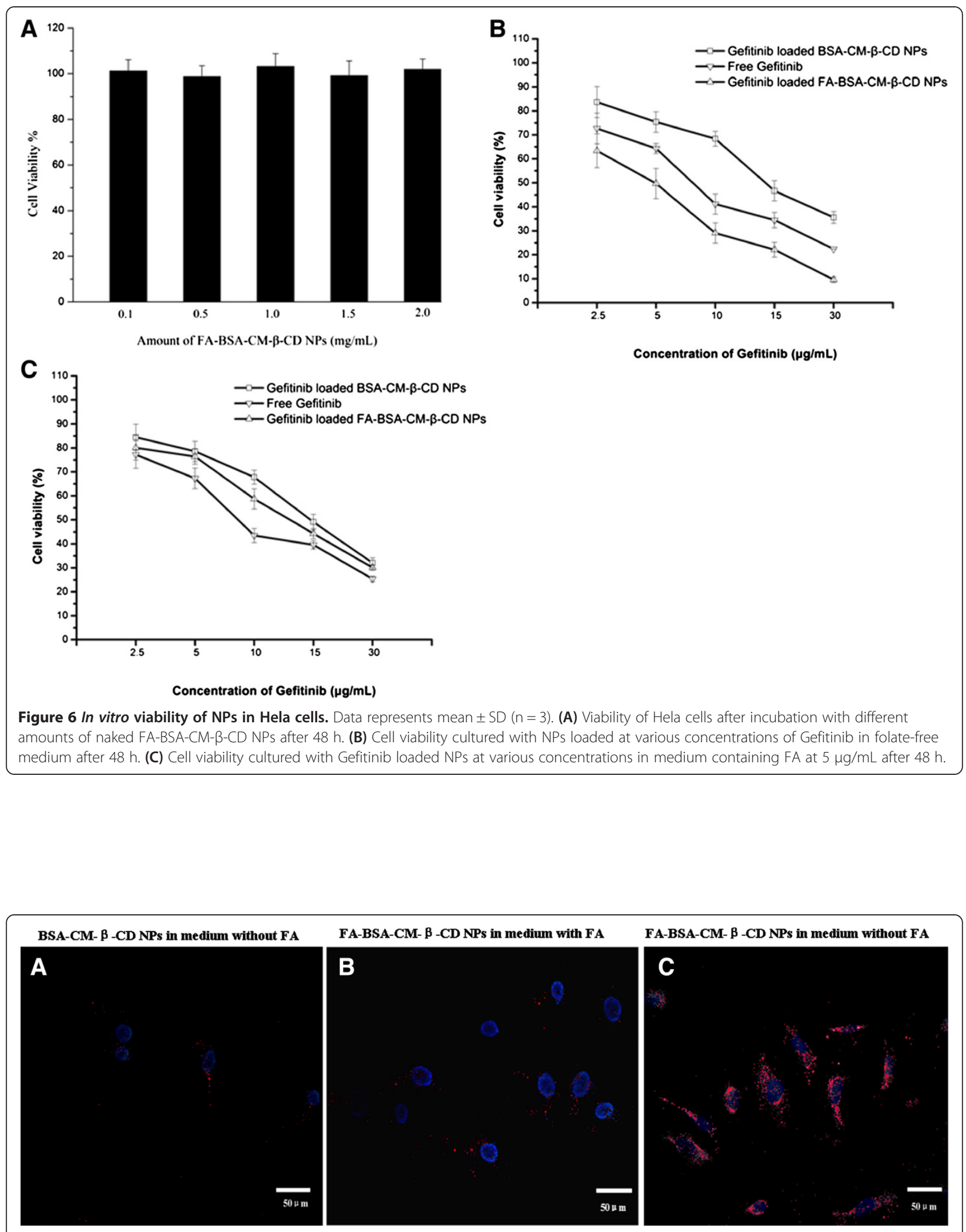

Figure 7 In vitro uptake ability for NPs. Fluorescent image of the uptake of BSA-CM- $\beta$-CD NPs in medium without FA (A). Fluorescence image of the uptake of FA-BSA-CM- $\beta-C D$ NPs in medium with FA (B) and without FA (C). 


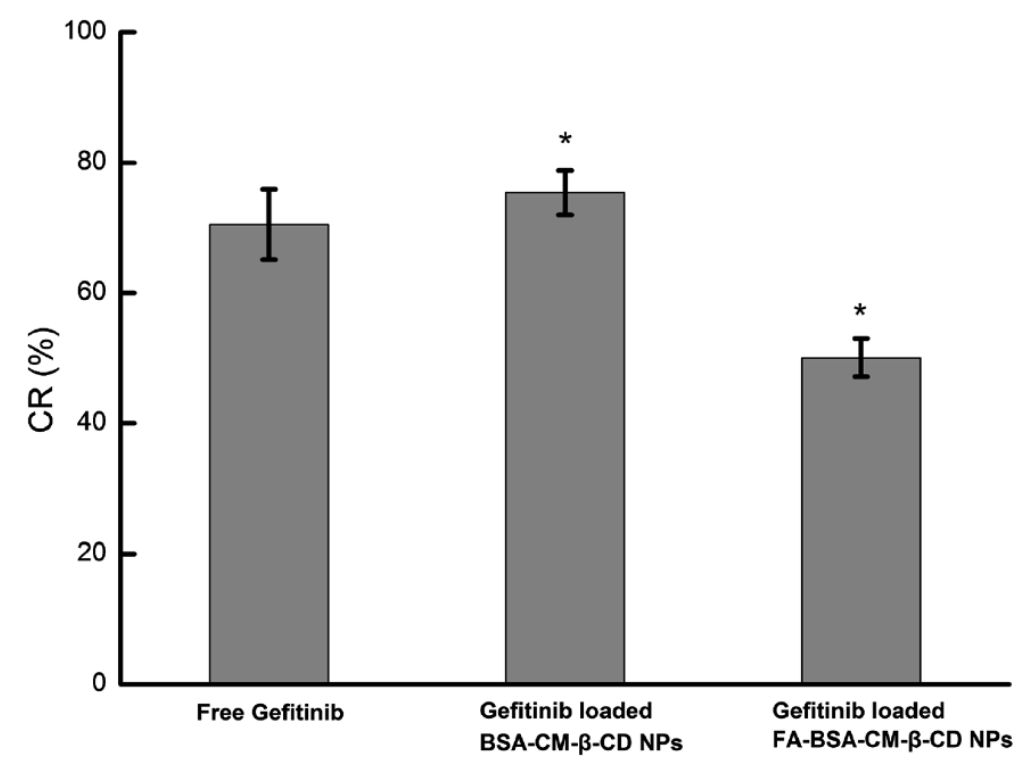

Figure 8 Results of intracellular ATP level assay after $48 \mathrm{~h}$ incubation with different preparations. Data were expressed as mean \pm SD $(n=3) .{ }^{*} P<0.05$, vs the free Gefitinib group.

reported that different conjugation with targeting ligands, such as iRGD, siRNA and disaccharide, could enhance uptake or change the endocytosis pathway of NPs resulting in improving cytotoxicity to cancer cells [38-41]. The results demonstrated that both NPs were internalized into cells mainly depending on clathrinmediated endocytosis and macropinocytosis being proved by the significant uptake reduction of both NPs in treated cells with chlorpromazine and cytochalasin D. In contrast, the regulation of caveolae-mediated endocytosis on NPs internalization was not significantly different from untreated group.

\section{Conclusions}

In summary, $C M-\beta-C D$ was conjugated with BSA by carbodiimide coupling. FA as a small targeting molecule, was bond to the surface of NPs. Gefitinib loaded FA-BSA-CM$\beta$-CD NPs showed good monodispersity, negative charge and homogenous particle size. The encapsulating efficiency of Gefitinib and the release pattern were investigated in vitro. MTT results showed that no obvious cytotoxity was observed when incubating naked FABSA-CM- $\beta-C D$ NPs with Hela cells. The free folic acid competition study showed that the cell inhibition of FA conjugated NPs in FR positive cells was significantly enhanced
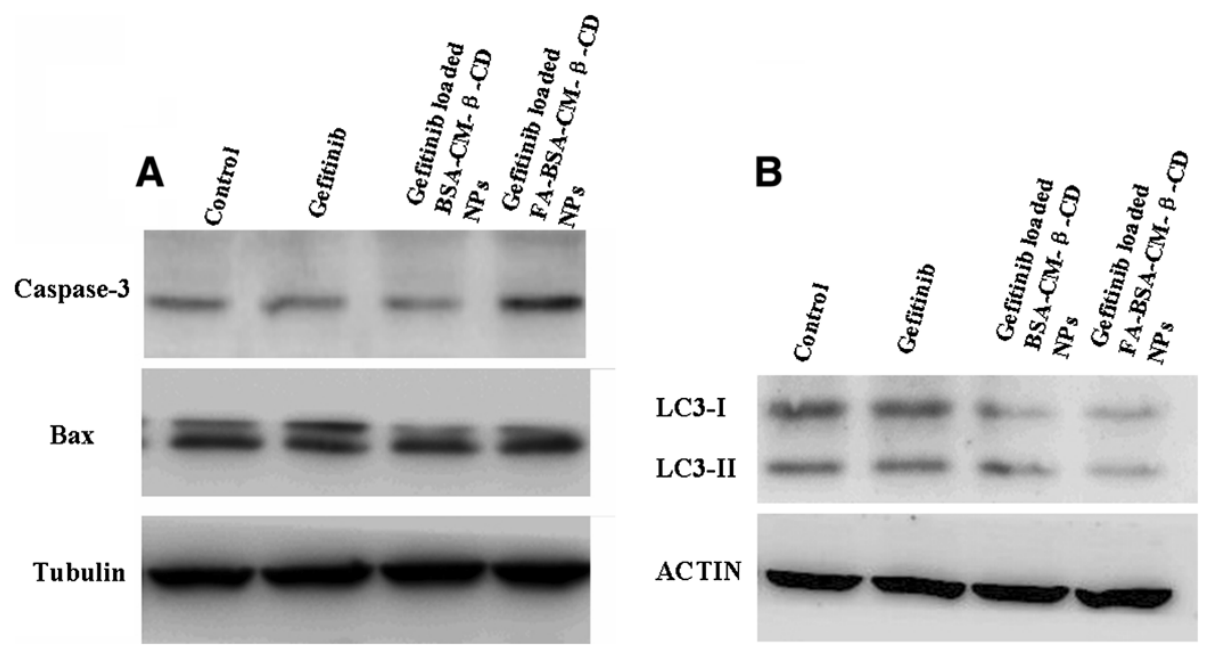

Figure 9 Apoptotic effects of various Gefitinib formulations on Hela cells. (A) Western blot analysis of the expression levels of Bax and caspase-3 proteins in Hela cells after treatments. (B) Western blot analysis of the expression levels of LC3 proteins in Hela cells after treatments. 


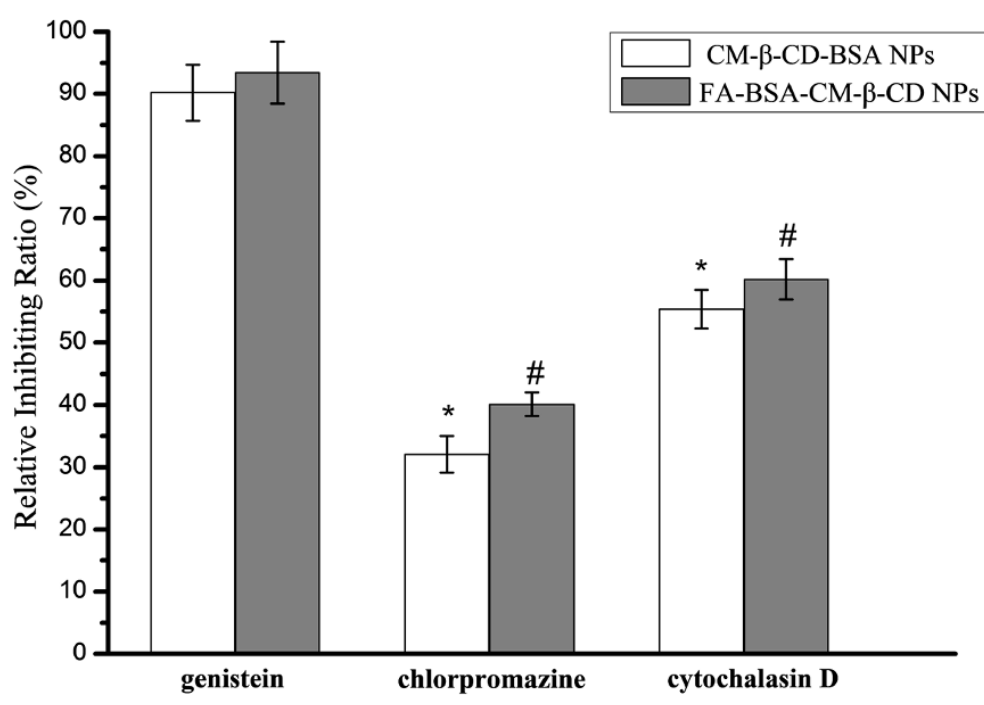

Figure 10 Effects of endocytic inhibitors on the uptaking ability of the two NPs in Hela cells. ${ }^{*} P<0.05$, vs the BSA-CM- $\beta$-CD NPs group treated with genistein in Hela cells, ${ }^{P} P<0.05$, vs the FA-BSA-CM- $\beta$-CD NPs group treated with genistein in Hela cells.

when FA was removed from the medium. Gefitinib loaded FA-BSA-CM- $\beta$-CD NPs inhibited cells autophagy by down-regulating LC3, and promoted apoptosis of Hela cells through the prevention of ATP generation and elevating the expression of caspase- 3 protein. It also confirmed that clathrin-mediated endocytosis and macropinocytosis played an important role on the internalization of both NPs. Taken together, FA-BSA$C M-\beta-C D$ NPs presented the promising candidates as a folate receptor-positive tumor-targeting carrier via folate mediation.

\section{Methods \\ Materials}

BSA was purchased from Sigma (USA), Carboxymethyl$\beta$-Cyclodextrin Sodium Salt $(C M-\beta-C D)$ was purchased from Zhiyuan Bio-Technology Co., Ltd (China), 1-ethyl3-(3-dimethylaminopropyl) carbodiimide (EDAC) and folic acid were obtained from Sigma Chemicals (St Louis, US). Gefitinib was purchased from Eastbang Pharmaceutical Co., Ltd (China). All other chemicals were of reagent grade and were used as received.

\section{Synthesis of BSA-CM- $\beta-C D$ conjugates}

$8.0 \mathrm{mg}$ of $C M-\beta-C D$ was dissolved in $8.0 \mathrm{~mL}$ PBS buffer solution ( $\mathrm{pH}$ 5.8). Then, the solution was added into a centrifuge tube in the presence of $40.0 \mathrm{mg}$ EDC and $16.0 \mathrm{mg}$ NHS. After the tube was rotated constantly for $1 \mathrm{~h}, 8.0 \mathrm{mg}$ BSA were added into the tube. Subsequently, the tube was rotated overnight. Finally, the BSA-CM$\beta$-CD conjugates were collected by dialysis filter with a dialysis bag with 1000 molecular weight cutoff to remove the uncoupled CM- $\beta-C D$.

\section{Preparation of Gefitinib loaded FA-BSA-CM- $\beta-C D$ self-assembled nanoparticles}

$20 \mathrm{mg}$ conjugates were suspended in $20 \mathrm{~mL}$ of distilled water to achieve a solution with concentration of $1 \mathrm{mg} / \mathrm{mL}$. Drug solution $(0.4 \mathrm{mg} / \mathrm{mL})$ was prepared by $2 \mathrm{mg}$ Gefitinib was dissolved in $5 \mathrm{ml}$ dichloromethane. Gefitinib loaded BSA-CM- $\beta$-CD NPs were prepared by quickly dropping $5.0 \mathrm{~mL}$ of drug solution into $20 \mathrm{~mL}$ distilled water containing $20 \mathrm{mg}$ conjugates at $30^{\circ} \mathrm{C}$ under continuously stirring for $24 \mathrm{~h}$ to remove dichloromethane. FA was conjugated with BSA-CM- $\beta$-CD NPs as the following: $5 \mathrm{mg}$ folic acid was dissolved in $10 \mathrm{~mL}$ phosphate buffer suspension (pH 7.4), then $15.0 \mathrm{mg}$ EDC and 5.0 mg NHS were added under stirring constantly for $1 \mathrm{~h}$. Entrapment efficiency of FA conjugated with BSA-CM- $\beta$-CD NPs was determined as the ratio of actual FA loading amount to the initial added FA amount. The solution was centrifuged at $16,000 \mathrm{rpm}$ for $30 \mathrm{~min}$. NPs collected were washed 3-4 times with deionized water and centrifuged at 16,000 rpm for $20 \mathrm{~min}$, freeze drying to obtain powders.

Characterization of Gefitinib loaded FA-BSA-CM- $\beta-C D$ NPs

The characterization of Gefitinib loaded FA-BSA-CM- $\beta-$ CD NPs was investigated by using AFFINITY-1 IR spectroscopy (Shimadzu, Kyoto, Japan). Its morphology was observed by using transmission electron microscope (TEM) (JEM-1200EX, Tokyo, Japan) and the mean diameter and zeta potential were determined by Zetasizer (Nano ZS90, Malvern, UK). The encapsulation efficiency (EE) of Gefitinib 
in FA-BSA-CM- $\beta-C D$ NPs was calculated using the equation listed below.

$$
\mathrm{EE}(\%)=\frac{\text { Weight of initially added drug-Weight of free drug in supernatant }}{\text { Weight of initially added drug }} \times 100
$$

\section{Assessment of drug release}

The drug releases were carried out in PBS containing $10 \%$ serum with different $\mathrm{pH}$ at $37.0 \pm 0.5^{\circ} \mathrm{C}$ under genthe agitation. $10 \mathrm{~mL} \mathrm{PBS}(\mathrm{pH}$ 7.4) in which accurate weighed $10 \mathrm{mg}$ dried Gefitinib-loaded FA-BSA-CM- $\beta$ CD NPs were suspended was put into a dialysis bag with 1000 molecular weight cutoff and the dialysis bag was immersed into $100 \mathrm{~mL}$ phosphate buffer solution containing $10 \%$ serum maintained at $\mathrm{pH} 7.4$ or 5.0 at $37.0 \pm 0.5^{\circ} \mathrm{C}$. At predetermined intervals, $5 \mathrm{~mL}$ of release medium was withdrawn and the same volume of fresh buffer solution was added. Samples were filtered through $0.45 \mu \mathrm{m}$ filter and the concentrations of Gefitinib released were analyzed by spectrophotometry at $338 \mathrm{~nm}$.

\section{Cell viability assays}

A 3-(4,5-dimethylthiazol-2-yl)-2,5- diphenyltetrazolium bromide (MTT) assay was used to investigate cell viability. Hela cells were chosen and used as a model folate receptor-positive cell line in angiogenesis targeting delivery and treatment for their high FR expression $[42,43]$. Hela cells were seeded into the 96-well plate at a density of $5 \times 10^{4} / \mathrm{mL}$ and attached for $24 \mathrm{~h}$ at $37^{\circ} \mathrm{C}$ in both folate-free medium and the medium containing folate at $5 \mu \mathrm{g} / \mathrm{mL}$ under $5 \% \mathrm{CO}_{2}$. Then, the cells were treated with free Gefitinib and Gefitinib-loaded NPs for $48 \mathrm{~h}$, followed by addition of $20 \mu \mathrm{L}$ MTT $(5 \mathrm{mg} / \mathrm{mL})$ and incubated for $4 \mathrm{~h}$ at $37^{\circ} \mathrm{C}$. Then, the supernatant was carefully removed and $150 \mu \mathrm{L}$ DMSO was added to each well and stirred for $30 \mathrm{~min}$. The absorbance was measured using microplate reader at $490 \mathrm{~nm}$.

\section{Uptaking ability of different kinds of NPs in Hela cells}

Hela cells, (a folate receptor-positive cell line) a model cell line, were applied to investigate cell uptake ability of different kinds of nanoparticles. Rhodamine B as the fluorescent marker was encapsulated in NPs. Cells were cultured in flasks supplemented with both folate-free medium and the medium containing folate $5 \mu \mathrm{g} / \mathrm{mL}$ at $37^{\circ} \mathrm{C}$ and $5 \% \mathrm{CO}_{2}$. When the cell concentrations reached $5 \times 10^{4} / \mathrm{mL}, 100 \mu \mathrm{L}$ of medium containing cells was transferred to the 96-well plate and treated with different Rhodamine B-labeled nanoparticles. At specified time, NPs were withdrawn and the wells were washed with PBS. Nucleus was stained with Hoechst for 15 min at $37^{\circ} \mathrm{C}$ followed by double washing with PBS. The internalization of RhB-loaded NPs into cells was observed using fluorescent microscopy (DMI400B, Leica, Germany).

\section{Intracellular ATP level assay}

Free Gefitinib, Gefitinib loaded BSA-CM- $\beta-C D$ NPs, Gefitinib loaded FA- BSA-CM- $\beta$-CD NPs at the same drug concentration were added into the 96-well plate filled with Hela cells at a density of $5 \times 10^{4} / \mathrm{mL}$ for $48 \mathrm{~h}$. The luciferin-luciferase-based ATP luminescence assay kit was applied to determine the changing rates of intracellular ATP level (CR\%) calculated using equation below.

$$
\begin{aligned}
\mathrm{CR}(\%) & =\frac{\text { ATP level of Hela cells treated with free drug or NPs }}{\text { ATP level of untreated Hela cells }} \\
& \times 100
\end{aligned}
$$

\section{Tracking of uptake pathways using various endocytic inhibitors}

In order to analyze the potential mechanism on uptake pathways of nanoparticles, three types of endocytic inhibitors including cytochalasin $\mathrm{D}(30 \mu \mathrm{M}$, macropinocytosis), genistein $(1 \mu \mathrm{g} / \mathrm{mL}$, caveolae mediated endocytosis) and chlorpromazine $(10 \mu \mathrm{g} / \mathrm{mL}$, clathrin mediated endocytosis) were preincubated with Hela cells in 96-well plate for $30 \mathrm{~min}$, respectively. Then both FITC labeled BSA-CM- $\beta$ CD NPs and FA- BSA-CM- $\beta-C D$ NPs were treated with cells to track the uptake pathways. The effects of various inhibitors on the uptake pathway of the NPs were evaluated by comparing the intracellular fluorescent intensity between treatment of adding inhibitors and non-inhibitors.

\section{Western blot assay}

After treated with free Gefitinib, Gefitinib loaded BSACM- $\beta-C D$ NPs, Gefitinib loaded FA-BSA-CM- $\beta-C D$ NPs, cells were harvested, washed twice with ice cold PBS, then lysed in RIPA buffer (150 mM NaCl, 1\% NP-40, 1\% SDS, $1 \mathrm{mM}$ PMSF, $10 \mu \mathrm{g} / \mathrm{mL}$ leupeptin, $1 \mathrm{mM}$ aprotinin, $50 \mathrm{mM}$ Tris-Cl, $\mathrm{pH}$ 7.4). The cell lysate was cleared by centrifugation at $12,000 \times \mathrm{g}$ for $25 \mathrm{~min}$. Cell lysate containing $50 \mu \mathrm{g}$ protein in $20 \mu \mathrm{L}$ was separated by $10 \%$ SDS-PAGE and the protein was transferred onto polyvinylidene fluoride (PVDF) membrane. After blocking with $1 \%$ BSA, the PVDF membrane was incubated with the primary antibodies (caspase-3, Bax, tubulin, LC3) at $4^{\circ} \mathrm{C}$ overnight. Subsequently, incubated with appropriate 
secondary antibody for $1 \mathrm{~h}$ and stained with ECL. The level of the targeted proteins were photographed and analyzed by UVP gel analysis system.

\section{Abbreviations}

FA: Folic acid; FR: Folate receptor; BSA: Bovine Serum Albumin; CM- $\beta$ -

CD: Carboxymethyl- $\beta$-Cyclodextrin; NPs: Nanoparticles.

\section{Competing interests}

The authors declare that they have no competing interests.

\section{Authors' contributions}

YS and CS performed the preparation and characteristics of various kinds of NPs and co- drafted the manuscript. LZ supervised the whole work. WC, LL, $\mathrm{BF}$ and $\mathrm{ML}$ helped with the biological study. HL and RS helped in the analysis of biological data. All authors read and approved the final manuscript.

\section{Acknowledgements}

This work is supported by Foundation of Liaoning Educational Committee (No. L2014339) and Innovative Program of University Students of Liaoning Province (No. 201410160008).

\section{Author details}

${ }^{1}$ College of Pharmacy, Liaoning Medical University, Jinzhou 121000, P R China. ${ }^{2}$ College of Veterinary Medicine, Liaoning Medical University, Jinzhou 121000 P R China. ${ }^{3}$ National Vaccine \& Serum Institute, Beijing 100024, China. ${ }^{4}$ Central Laboratory of Liaoning Medical University, Jinzhou 121000, P R China.

Received: 17 September 2014 Accepted: 12 October 2014 Published online: 30 October 2014

\section{References}

1. lafisco M, Delgado-Lopez JM, Varoni EM, Tampieri A, Rimondini L, Gomez-Morales J, Prat M: Cell Surface Receptor Targeted Biomimetic Apatite Nanocrystals for Cancer Therapy. Small 2013, 9(22):3834-3844.

2. Chen H, Zhao Y, Wang H, Nie G, Nan K: Co-delivery strategies based on multifunctional nanocarriers for cancer therapy. Curr Drug Metab 2012, 13(8):1087-1096

3. Danhier F, Feron O, Préat $\mathrm{V}$ : To exploit the tumor microenvironment: Passive and active tumor targeting of nanocarriers for anti-cancer drug delivery. J Control Release 2010, 148(2):135-146.

4. Torchilin VP: Multifunctional nanocarriers. Adv Drug Deliv Rev 2012, 64:302-315.

5. Gao H, Zhang Q, Yu Z, He Q: Cell-penetrating peptide-based intelligent liposomal systems for enhanced drug delivery. Curr Pharm Biotechnol 2014, 15:210-219.

6. Mattheolabakis G, Taoufik E, Haralambous S, Roberts ML, Avgoustakis K: In vivo investigation of tolerance and antitumor activity of cisplatin-loaded PLGA-mPEG nanoparticles. Eur J Pharm Biopharm 2009, 71(2):190-195.

7. Hyung Park J, Kwon S, Lee M, Chung H, Kim JH, Kim YS, Park RW, Kim IS, Bong Seo S, Kwon IC, Young Jeong S: Self-assembled nanoparticles based on glycol chitosan bearing hydrophobic moieties as carriers for Gefitiniborubicin: in vivo biodistribution and anti-tumor activity. Biomaterials 2006, 27(1):119-126.

8. Yu Z, Schmaltz RM, Bozeman TC, Paul R, Rishel MJ, Tsosie KS, Hecht SM: Selective Tumor Cell Targeting by the Disaccharide Moiety of Bleomycin. J Am Chem Soc 2013, 135:2883-2886.

9. William P, Vincent M, Maureen Z, Jennifer D, Katerina P, Inderpal S, Bhuvanesh S, Robert H, Valerie R, Lucinda F, Elaine M, Doris K, Richard W, Mark K, Harold V: EGF receptor gene mutations are common in lung cancers from "never smokers" and are associated with sensitivity of tumors to gefitinib and erlotinib. Proc Natl Acad Sci USA 2004, 101(36):13306-13311.

10. Morabito A, Costanzo R, Rachiglio AM, Pasquale R, Sandomenico C, Franco R, Montanino A, De Lutio E, Rocco G, Normanno N: Activity of Gefitinib in a Non-Small-Cell Lung Cancer Patient with Both Activating and Resistance EGFR Mutations. J Thorac Oncol 2013, 8(7):e59-e60.

11. Ozao-Choy J, Ma G, Kao J, Wang GX, Meseck M, Sung M, Schwartz M, Divino CM, Pan PY, Chen SH: The novel role of tyrosine kinase inhibitor in the reversal of immune suppression and modulation of tumor microenvironment for immune-based cancer therapies. Cancer Res 2009, 69(6):2514-2522.
12. Sordella R, Bell DW, Haber DA, Settleman J: Gefitinib-sensitizing EGFR mutations in lung cancer activate anti-apoptotic pathways. Science 2004, 305(5687):1163-1167.

13. Cohen MH, Williams GA, Sridhara R, Chen G, McGuinn WD, Morse D, Abraham S, Rahman A, Liang C, Lostritto R, Baird A, Pazdur R: United States Food and Drug Administration drug approval summary gefitinib (ZD1839; Iressa) tablets. Clin Cancer Res 2004, 10(4):1212-1218.

14. Kakuta T, Takashima Y, Nakahata M, Otsubo M, Yamaguchi H, Harada A: Preorganized Hydrogel: Self-Healing Properties of Supramolecular Hydrogels Formed by Polymerization of Host-Guest-Monomers that Contain Cyclodextrins and Hydrophobic Guest Groups. Adv Mater 2013, 25(20):2849-2853.

15. Loftsson T, Brewster ME: Cyclodextrins as functional excipients: methods to enhance complexation efficiency. J Pharm Sci 2012, 101(9):3019-3032.

16. Loftsson T, Duchêne D: Cyclodextrins and their pharmaceutical applications. Int J Pharm 2007, 329:1-11.

17. Kurkov SV, Loftsson T: Cyclodextrins. Int J Pharm 2013, 453(1):167-180

18. Szente L, Szemán J: Cyclodextrins in Analytical Chemistry: Host-Guest Type Molecular Recognition. Anal Chem 2013, 85(17):8024-8030.

19. Del Valle EM: Cyclodextrins and their uses: a review. Process Biochem 2004, 39(9):1033-1046.

20. Agasti SS, Liong M, Tassa C, Chung HJ, Shaw SY, Lee H, Weissleder R: Supramolecular host-guest interaction for labeling and detection of cellular biomarkers. Angew Chem Int Ed Engl 2012, 51(2):450-454.

21. Dorokhin D, Hsu SH, Tomczak N, Reinhoudt DN, Huskens J, Velders AH, Vancso GJ: Fabrication and luminescence of designer surface patterns with $\beta$-cyclodextrin functionalized quantum dots via multivalent supramolecular coupling. ACS Nano 2010, 4:137-142.

22. Zhang B, Jin H, Li Y, Chen B, Liu S, Shi D: Bioinspired synthesis of gadolinium-based hybrid nanoparticles as MRI blood pool contrast agents with high relaxivity. J Mater Chem 2012, 22(29):14494-14501.

23. Sun SK, Dong $L$, Cao Y, Sun $H$, Yan X: Fabrication of multifunctional Gd2O3/Au hybrid nanoprobe via a one-step approach for near-infrared fluorescence and magnetic resonance multimodal imaging in vivo. Anal Chem 2013, 85(17):8436-8441.

24. Zhang B, Li Q, Yin P, Rui Y, Qiu Y, Wang Y, Shi D: Ultrasound-triggered BSA/SPION hybrid nanoclusters for liver-specific magnetic resonance imaging. ACS Appl Mater Interfaces 2012, 4(12):6479-6486.

25. Zhang $B$, Wang $X$, Liu F, Cheng Y, Shi D: Effective reduction of nonspecific binding by surface engineering of quantum dots with bovine serum albumin for cell-targeted imaging. Langmuir 2012, 28(48):16605-16613.

26. Cagle PT, Zhai QJ, Murphy L, Low PS: Folate receptor in adenocarcinoma and squamous cell carcinoma of the lung: potential target for folatelinked therapeutic agents. Arch Pathol Lab Med 2013, 137(2):241-244.

27. Chen C, Ke J, Zhou XE, Yi W, Brunzelle JS, Li J, Yong EL, Xu HE, Melcher K: Structural basis for molecular recognition of folic acid by folate receptors. Nature 2013, 500(7463):486-489.

28. Leamon CP: Folate-targeted drug strategies for the treatment of cancer Curr Opin Investig Drugs 2008, 9(12):1277-1286.

29. Coney LR, Tomassetti A, Carayannopoulos L, Frasca V, Kamen BA, Colnaghi MI, Zurawski VR Jr: Cloning of a tumor-associated antigen: MOv18 and MOv19 antibodies recognize a folate-binding protein. Cancer Res 1991, 51(22):6125-6132.

30. Weitman SD, Frazier KM, Kamen BA: The folate receptor in central nervous system malignancies of childhood. J Neurooncol 1994, 21(2):107-112.

31. Campbell IG, Jones TA, Foulkes WD, Trowsdale J: Folate-binding protein is a marker for ovarian cancer. Cancer Res 1991, 51(19):5329-5338.

32. Reddy JA, Low PS: Folate-mediated targeting of therapeutic and imaging agents to cancers. Crit Rev Ther Drug Carrier Syst 1998, 15(6):587-627.

33. Chen $\mathrm{H}$, Ahn R, Van den Bossche J, Thompson DH, O'Halloran TV: Folate-mediated intracellular drug delivery increases the anticancer efficacy of nanoparticulate formulation of arsenic trioxide. Mol Cancer Ther 2009, 8(7):1955-1963.

34. Chu BC, Kramer FR, Orgel LE: Synthesis of an amplifiable reporter RNA for bioassays. Nucleic Acids Res 1986, 14(14):5591-5603.

35. Ghosh SS, Kao PM, McCue AW, Chappelle HL: Use of maleimide-thiol coupling chemistry for efficient syntheses of oligonucleotide-enzyme conjugate hybridization probes. Bioconjug Chem 1990, 1(1):71-76.

36. Staros NV, Wright RW, Swingle DM: Enhancement by N-hydroxysulfosuccinimide of water-soluble carbodiimide-mediated coupling reactions. Anal Biochem 1986, 156(1):220-222. 
37. Khalil SK, El-Feky GS, El-Banna ST, Khalil WA: Preparation and evaluation of warfarin- $\beta$-cyclodextrin loaded chitosan nanoparticles for transdermal delivery. Carbohydr Polym 2012, 90(3):1244-1253.

38. Joo Kl, Xiao L, Liu S, Liu Y, Lee CL, Conti PS, Wong MK, Li Z, Wang P: Crosslinked multilamellar liposomes for controlled delivery of anticancer drugs. Biomaterials 2013, 34(12):3098-3109.

39. Schroeder BR, Ghare MI, Bhattacharya C, Paul R, Yu Z, Zaleski PA, Bozeman TC, Rishel MJ, Hecht SM: The disaccharide moiety of bleomycin facilitates uptake by cancer cells. J Am Chem Soc 2014, 136(39):13641-13656.

40. Qi R, Liu S, Chen J, Xiao H, Yan L, Huang Y, Jing X: Biodegradable copolymers with identical cationic segments and their performance in siRNA delivery. J Control Release 2012, 159(2):251-260.

41. Xiao H, Qi R, Liu S, Hu X, Duan T, Zheng Y, Huang Y, Jing X: Biodegradable polymer - cisplatin(IV) conjugate as a pro-drug of cisplatin(II). Biomaterials 2011, 32(30)::7732-7739.

42. Song Y, Shi W, Chen W, Li X, Ma H: Fluorescent carbon nanodots conjugated with folic acid for distinguishing folate-receptor-positive cancer cells from normal cells. J Mater Chem 2012, 22(25):12568-12573.

43. Feng D, Song Y, Shi W, Li X, Ma H: Distinguishing Folate-Receptor-Positive Cells from Folate-Receptor-Negative Cells Using a Fluorescence Off-On Nanoprobe. Anal Chem 2013, 85(13):6530-6535. enhance drug delivery and attenuate autophagy in folate receptor-positive cancer cells. Journal of Nanobiotechnology 2014 12:43.

\section{Submit your next manuscript to BioMed Central and take full advantage of:}

- Convenient online submission

- Thorough peer review

- No space constraints or color figure charges

- Immediate publication on acceptance

- Inclusion in PubMed, CAS, Scopus and Google Scholar

- Research which is freely available for redistribution 